\title{
Recuperação Pós-Operatória de Sangue em Doentes Submetidos a Artroplastias Totais do Joelho ou da Anca
}

\author{
Postoperative Recovery of Blood in Patients Undergoing \\ Total Knee or Hip Arthroplasty
}

\author{
Hugo LARANJEIRA ${ }^{1,4}$, Celeste COIMBRA ${ }^{2}$, Nuno FERNANDES ${ }^{3}$, Romeu PINHO ${ }^{2}$, Rui ANJOS ${ }^{1}$, Elisabeth MESQUITA ${ }^{1}$, \\ António BARROS ${ }^{4}$, Rita FERREIRA4 ${ }^{4}$, Lúcia BORGES ${ }^{1}$ \\ Acta Med Port 2013 Sep-Oct;26(5):511-516
}

\section{RESUMO}

Introdução: Na artroplastia total do joelho e na artroplastia total da anca ocorrem perdas de sangue consideráveis, sendo necessário recorrer frequentemente a transfusões de concentrado de eritrócitos. No entanto, para além dos potenciais riscos inerentes, o sangue homólogo é um recurso limitado e caro. No sentido de reduzir o recurso a transfusões homólogas, tem-se desenvolvido estratégias alternativas, como a recuperação pós-operatória de sangue.

Objetivos: Avaliação da implementação da recuperação pós-operatória de sangue no consumo de concentrado de eritrócitos no período pós-operatório e no tempo de internamento, pela avaliação retrospetiva de doentes submetidos a artroplastia total do joelho ou a artroplastia total da anca.

Material e Métodos: Os 976 doentes foram divididos em 2 grupos: CELL TRANS - doentes submetidos à intervenção após a implementação da recuperação pós-operatória de sangue e CONT - submetidos à intervenção antes da implementação da estratégia referida. O número de unidades de concentrado de eritrócitos administradas foi obtido pela análise das requisições ao Serviço de Imunohemoterapia e o tempo de internamento foi obtido através do Sistema de Apoio ao Médico. A análise estatística foi realizada com recurso à linguagem $R$, considerando-se diferenças significativas entre os grupos quando $p<0,05$.

Resultados: Após a introdução da recuperação pós-operatória de sangue, $29 \%$ dos doentes submetidos a artroplastia total do joelho e $38 \%$ dos doentes submetidos a artroplastia total da anca receberam concentrado de eritrócitos no período pós-operatório, um número consideravelmente menor ao observado antes da implementação da estratégia, $68 \%$ e $59 \%$ respetivamente. Concomitantemente, verificou-se uma redução estatisticamente significativa no tempo de internamento de 9,0 para 8,3 dias para o caso dos doentes submetidos a artroplastia total do joelho e de 9,1 para 8,8 dias para o caso dos doentes submetidos a artroplastia total da anca.

Conclusões: A recuperação pós-operatória de sangue em doentes submetidos a artroplastia total do joelho ou a artroplastia total da anca reduz significativamente a necessidade de transfusão de concentrado de eritrócitos bem como o tempo de internamento.

Palavras-chave: Artroplastia da Anca; Artroplastia do Joelho; Cuidados Pós-Operatórias; Perda de Sangue durante a Cirurgia.

\section{ABSTRACT}

Background: In total knee arthroplasty and total hip arthroplasty occurs a considerable loss of blood, which often requires homologous transfusions of red blood cells. However, homologous transfusions have risks, besides being expensive and a limited resource. Thus, in order to reduce the need for homologous transfusions alternatives strategies have been developed, like post-operative recovery of blood.

Objectives: Evaluation of post-operative recovery of blood in postoperatively red blood cells consumption and in hospital stay, by a retrospective analysis of patients undergoing total knee arthroplasty or total hip arthroplasty.

Material and Methods: Patients $(n=976)$ were divided in 2 groups: CELL TRANS - after implementation of postoperative recovery of blood and CONT - before implementation of the strategy. The red blood cells requests to Serviço de Imunohemoterapia gave the number of RBC units administered and the length of hospital stay was obtained through the Sistema de Apoio ao Médico. Statistical analysis was performed using the $\mathrm{R}$ language, considering significant differences between groups when $p$ values $<0,05$.

Results: After the introduction of this strategy, $29 \%$ of patients undergoing total knee arthroplasty and $38 \%$ of patients undergoing total hip arthroplasty received red blood cells in the postoperative period, a number considerably lower to that observed before the implementation of post-operative recovery of blood, $68 \%$ and $59 \%$ respectively. Concomitantly, there was a statistically significant reduction in the length of stay of 9.0 to 8.3 days in the case of total knee arthroplasty patients and from 9.1 to 8.8 days in the case of patients undergoing total hip arthroplasty.

Conclusion: Post-operative recovery of blood in patients undergoing total knee arthroplasty or total hip arthroplasty significantly reduces the need for red blood cells transfusion and the length of hospital stay.

Keywords: Arthroplasty, Replacement, Hip; Arthroplasty, Replacement, Knee; Blood Loss, Surgical; Postoperative Care.

\section{INTRODUÇÃO}

As cirurgias ortopédicas, como é o caso da artroplastia total do joelho (ATJ) e da artroplastia total da anca (ATA) têm vindo a aumentar nas sociedades modernas, em parte devido ao aumento da longevidade e consequente perda de qualidade do tecido ósseo. ${ }^{1,2}$ Estas cirurgias estão associadas a uma perda significativa de sangue ${ }^{3,4}$ pelo que a transfusão sanguínea é uma prática comum. ${ }^{5}$ Efetivamente, $20 \%$ a $50 \%$ dos doentes submetidos a AT ${ }^{6,7}$ e $30 \%$ a

1. Serviço de Imunohemoterapia. Centro Hospitalar do Baixo Vouga, E.P.E. Aveiro. Portugal.

2. Serviço de Ortopedia. Centro Hospitalar do Baixo Vouga, E.P.E. Aveiro. Portugal.

3. Serviço de Anestesiologia. Centro Hospitalar do Baixo Vouga, E.P.E. Aveiro. Portugal.

4. Departamento de Química. Universidade de Aveiro. Aveiro. Portugal.

Recebido: 23 de Dezembro de 2012 - Aceite: 19 de Julho de 2013 | Copyright $\odot$ Ordem dos Médicos 2013 
$40 \%$ dos doentes submetidos a ATA recebem transfusão de concentrado de eritrócitos (CE). ${ }^{8}$ Os doentes submetidos a este tipo de cirurgia são responsáveis por um consumo relativamente elevado de CE. No Reino Unido $10 \%$ de todas as unidades de CE administrados nos hospitais são consumidas por doentes ortopédicos. Desses, $40 \%$ são usados em doentes submetidos a ATJ ou a ATA. ${ }^{3}$

As transfusões de componentes de sangue homólogo, para além do custo financeiro elevado que comportam, não estão isentas de riscos, estando associadas a um aumento da morbilidade e da mortalidade. Os riscos associados à transfusão de componentes de sangue homólogo incluem riscos infeciosos e não infeciosos. Os riscos infeciosos incluem a transmissão de vírus, ${ }^{9,10}$ bactérias, ${ }^{11,12}$ parasitas $^{9,12}$ e ainda a possível transmissão de priões. ${ }^{13}$ Enquanto os riscos não infeciosos englobam reação transfusional hemolítica aguda, ${ }^{12,14}$ reação transfusional hemolítica tardia, ${ }^{12}$ reação febril não hemolítica, ${ }^{12}$ reação alérgica, ${ }^{12}$ lesão pulmonar aguda associada à transfusão, ${ }^{9,12}$ doença do enxerto contra o hospedeiro, ${ }^{12}$ imunossupressão, ${ }^{9}$ purpura pós-transfusional ${ }^{12}$ e erros de transfusão. ${ }^{9,15}$

Para além destes riscos associados à transfusão de componentes de sangue homólogo, o sangue homólogo constitui um recurso clínico valioso e a sua oferta é limitada. ${ }^{16,17}$ Nos últimos anos tem havido um aumento do uso de componentes de sangue homólogo por parte dos sistemas de cuidados de saúde dos países ocidentais. As estimativas sugerem um incremento de $6 \%$ por ano, o que resulta numa grave escassez de sangue homólogo e um aumento dos custos associados à sua aquisição. ${ }^{18}$

O interesse numa programação adequada das transfusões de componentes de sangue homólogo tem aumentado por várias razões, epidemiológicas, imunológicas, económicas e religiosas levando ao desenvolvimento de estratégias alternativas. ${ }^{19,20}$ De entre as estratégias alternativas à transfusão homóloga, a recuperação pós-operatória de sangue parece constituir uma boa alternativa em doentes submetidos a ATJ ${ }^{21,22}$ e a ATA, ${ }^{22,23}$ um tipo de cirurgias onde ocorre uma grande perda de sangue do local cirúrgico. ${ }^{6}$ A recuperação pós-operatória de sangue consiste na reinfusão de sangue recuperado do local cirúrgico, depois de filtrado, recorrendo a dispositivos específicos. ${ }^{24}$ Vários estudos sugerem que na prática clínica a utilização desta estratégia é segura. ${ }^{22,24-26}$

Este trabalho teve por objetivo, analisar o efeito da implementação da recuperação pós-operatória de sangue nas cirurgias ortopédicas ATJ e ATA no consumo de CE no período pós-operatório e no tempo de internamento.

\section{MATERIAL E MÉTODOS}

No presente estudo foram considerados 976 doentes submetidos às cirurgias ATJ e ATA, tendo sido acompanhados pelo Serviço de Ortopedia do Centro Hospitalar do Baixo Vouga, E.P.E. - Aveiro. Os doentes incluídos no estudo foram agrupados tendo em consideração a recuperação pós-operatória de sangue. Para o efeito avaliaram-se dois períodos temporais. Um período antes da implemen- tação da recuperação pós-operatória de sangue no Centro Hospitalar do Baixo Vouga, E.P.E. - Aveiro, em que foram analisados doentes submetidos às intervenções ATJ e ATA entre 1 de outubro de 2006 a 31 de dezembro de 2008. Estes doentes constituem o grupo CONT ( $n=230$ para ATJ e $n=266$ para ATA). Outro período após a implementação da recuperação pós-operatória de sangue utilizando o Cell Trans $^{\mathrm{TM}}$ (Summit Medical), em que foram analisados os doentes submetidos a ATJ e ATA entre 1 de junho de 2009 a 31 de dezembro de 2011, estes doentes constituem o grupo CELL TRANS ( $n=258$ para ATJ e $n=222$ para ATA). Os dados obtidos dos doentes submetidos a ATJ e dos doentes submetidos a ATA foram analisados separadamente.

O presente trabalho foi aprovado pela comissão de ética do Centro Hospitalar do Baixo Vouga, E.P.E. - Aveiro.

\section{Parâmetros considerados na recolha de dados dos doentes em estudo}

A informação relativa ao tempo de internamento de cada paciente foi obtido através do Sistema de Apoio ao Médico (SAM) enquanto o número de unidades de CE administradas a cada paciente no período pós-cirurgia foi obtido através da análise das requisições de sangue ao Serviço de Imuno-Hemoterapia do Centro Hospitalar do Baixo Vouga, E.P.E. - Aveiro. Os valores pré-operatórios de contagem de eritrócitos, concentração de hemoglobina e hematócrito foram obtidos através do Appolo (Sistema de informações para o laboratório de análises clínicas).

\section{Análise estatística}

Todos os cálculos foram realizados com recurso à linguagem $\mathrm{R}$ (http://www.r-project.org). ${ }^{27}$ As diferenças entre grupos foram consideradas significativas quando os valores de $p<0,05$.

\section{RESULTADOS}

\section{Caracterização da população em estudo}

De forma a caracterizar os grupos em estudo, foram avaliadas algumas características dos doentes, incluindo: a idade, as proporções relativas em termos de sexos e também os valores pré-operatórios de contagem de eritrócitos (Erit), concentração de hemoglobina $(\mathrm{Hb})$ e hematócrito (Ht) (Tabelas 1 e 2).

Como é possível constatar pela análise da Tabela 1 , os doentes submetidos a ATJ de ambos os grupos, CELL TRANS e CONT, não apresentaram diferenças estatisticamente significativas $(p>0,05)$ para nenhuma das características analisadas. As percentagens relativas de indivíduos do sexo masculino vs. feminino são também semelhantes entre os grupos CELL TRANS e CONT.

Para o caso dos doentes submetidos a ATA, não se observaram diferenças significativas entre os grupos CELL TRANS e CONT para as características analisadas ( $p>$ $0,05)$, à exceção da contagem de eritrócitos (Tabela 2 ) 
Tabela 1 - Características dos doentes em estudo submetidos a ATJ.

\begin{tabular}{|c|c|c|c|}
\hline & CELL TRANS & CONT & valor de $p$ \\
\hline Idade (anos) média \pm desvio padrão & $69,6 \pm 7,6$ & $68,8 \pm 7,4$ & 0,2107 \\
\hline Masculino / feminino (\%) & $29 / 71$ & $24 / 76$ & -- \\
\hline Erit $\left(\times 10^{12} / \mathrm{L}\right)$ média \pm desvio padrão & $4,42 \pm 0,46$ & $4,41 \pm 0,41$ & 0,8793 \\
\hline $\mathrm{Hb}(\mathrm{g} / \mathrm{dL})$ média \pm desvio padrão & $13,5 \pm 1,3$ & $13,4 \pm 1,2$ & 0,4943 \\
\hline Ht $(\%)$ média \pm desvio padrão & $40,5 \pm 4,3$ & $41,0 \pm 3,7$ & 0,5569 \\
\hline
\end{tabular}

Tabela 2 - Características dos doentes em estudo submetidos a ATA.

\begin{tabular}{cccc}
\hline & CELL TRANS & CONT & valor de $p$ \\
\hline Idade (anos) média \pm desvio padrão & $68,0 \pm 10,5$ & $66,1 \pm 11,4$ & 0,09639 \\
Masculino / feminino $(\%)$ & $45 / 55$ & $47 / 53$ & 0,0006952 \\
Erit $\left(\times 10^{12} / \mathrm{L}\right)$ média \pm desvio padrão & $4,38 \pm 0,46$ & $4,52 \pm 0,48$ & 0,8665 \\
$\mathrm{Hb}(\mathrm{g} / \mathrm{dL})$ média \pm desvio padrão & $13,6 \pm 1,4$ & $13,6 \pm 1,4$ & 0,9913 \\
$\mathrm{Ht}(\%)$ média \pm desvio padrão & $40,9 \pm 3,8$ & $41,5 \pm 4,3$ & 0
\end{tabular}

\section{Efeito da recuperação pós-operatória de sangue no consumo de CE}

Com o objetivo de determinar o efeito da implementação da recuperação pós-operatória de sangue, em doentes submetidos a ATJ ou a ATA comparou-se as unidades de CE consumidas por doentes do grupo CELL TRANS e do grupo CONT no período pós-operatório (Fig. 1).

Como é possível observar na Fig. 1, a frequência relativa de doentes que não recebeu nenhuma unidade CE é maior no grupo CELL TRANS em relação ao grupo CONT, tanto para os doentes submetidos a ATJ (Fig. 1A) como para os doentes submetidos a ATA (Fig. 1B), embora esta tendência seja mais acentuada no caso dos doentes submetidos a ATJ.

Adicionalmente determinou-se a proporção de doentes submetidos a ATJ e a ATA que receberam transfusão de pelo menos uma unidade de CE no período pós-operatório (Fig. 2).

No grupo CELL TRANS 29\% dos doentes submetidos a ATJ receberam pelo menos uma unidade de CE no período pós-operatório, uma percentagem consideravelmente mais baixa do que a observado no grupo CONT, que foi de $68 \%$ (Fig. 2A). No caso dos doentes submetidos a ATA, no grupo CELL TRANS a $38 \%$ dos doentes foi administrada pelo menos uma unidade de CE, percentagem consideravelmente menor do que o verificado para o grupo CONT, que foi de $59 \%$ (Fig. 2B).

\section{Efeito da recuperação pós-operatória de sangue no tempo de internamento}

No sentido de determinar o efeito da recuperação pós-operatória de sangue no tempo de internamento de doentes submetidos a ATJ ou a ATA, comparou-se o tempo de internamento do grupo CELL TRANS com o grupo CONT (Fig. 3).

Como se pode observar na Fig. 3A, para os doentes submetidos a ATJ, o tempo médio de internamento para os doentes do grupo CELL TRANS foi de 8,3 dias, enquanto para os doentes do grupo CONT foi de 9,0 dias, uma diferença entre os grupos estatisticamente significativa. Para os doentes submetidos a ATA, no grupo CELL TRANS verificou-se um tempo médio de internamento de 8,8 dias, valor significativamente menor do que o verificado no grupo CONT, como se pode observar na Fig. 3B.

\section{DISCUSSÃO}

Após a implementação da estratégia de recuperação pós-operatória de sangue, com recurso ao dispositivo Cell Trans $^{\mathrm{TM}}$, no Centro Hospitalar do Baixo Vouga, E.P.E. Aveiro, a frequência relativa de doentes submetidos a ATJ que não receberam nenhuma unidade de $C E$ aumentou consideravelmente (Fig. 1A). Nos doentes submetidos a ATA verificou-se uma tendência semelhante embora não tão acentuada (Fig. 1B). Os resultados estão de acordo com o descrito em outros estudos que avaliaram o efeito da mesma estratégia em cirurgias ortopédicas. .,28-31 $^{5}$ 
A

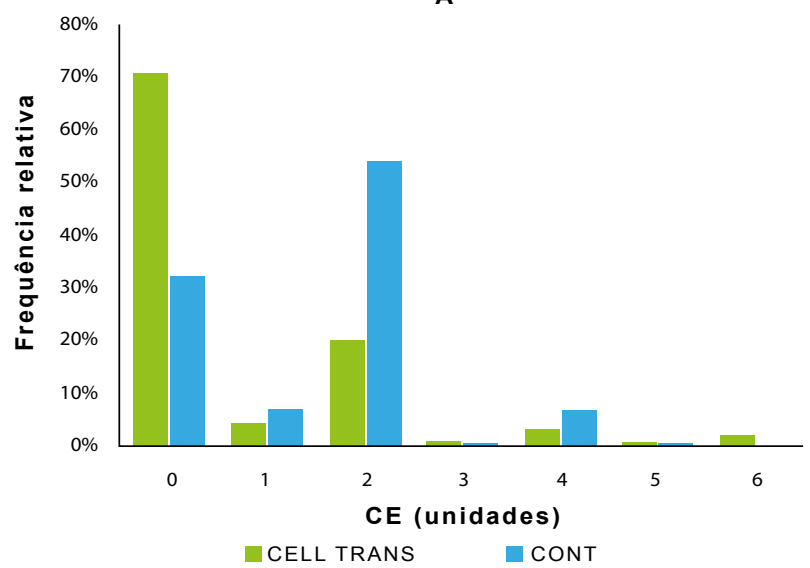

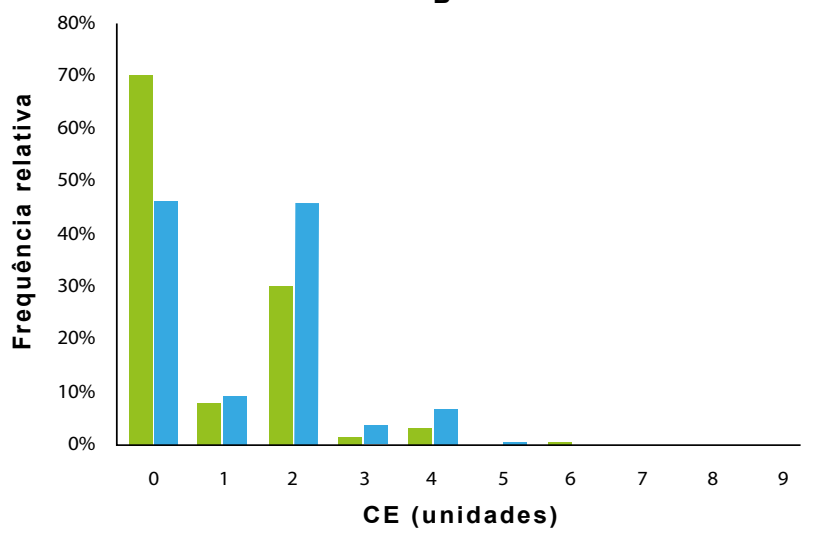

Figura 1 - A: Frequência relativa de doentes submetidos a ATJ que receberam CE; B: Frequência relativa de doentes submetidos a ATA que receberam $\mathrm{CE}$.

A

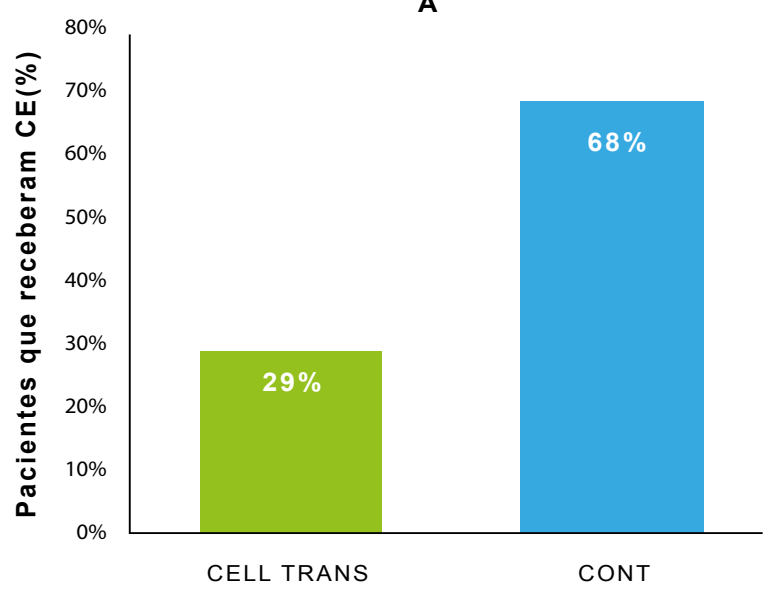

B

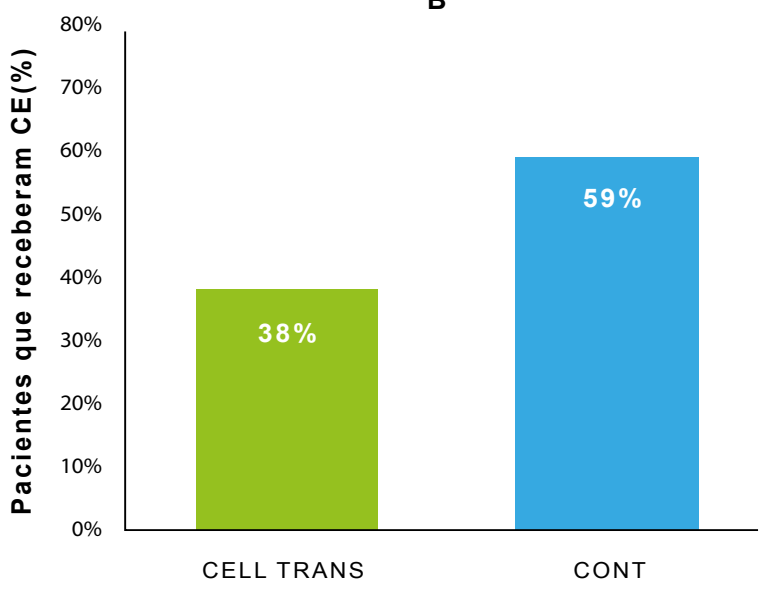

Figura 2 - A: Percentagem de doentes submetidos a ATJ que receberam pelo menos uma unidade de CE no período pós-operatório; B: Percentagem de doentes submetidos a ATA que receberam pelo menos uma unidade de CE no período pós-operatório.
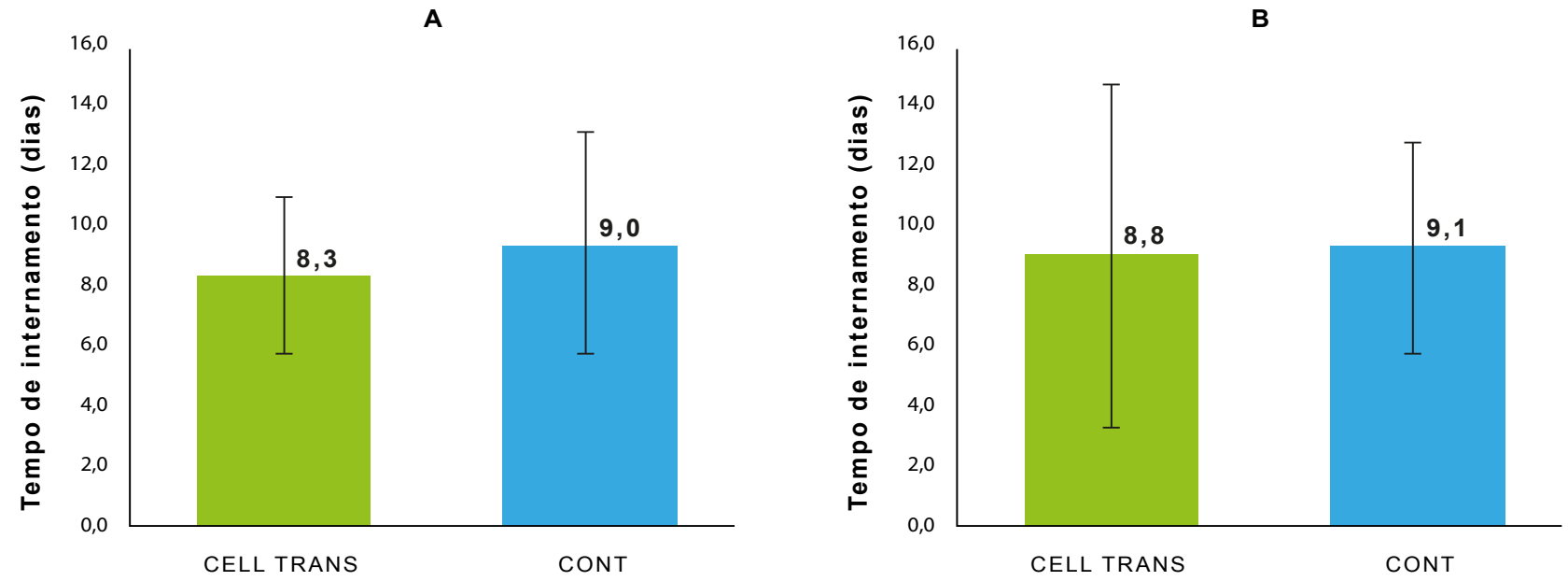

Figura 3 - A: Tempo de internamento dos doentes submetidos a ATJ. Os valores são apresentados como média \pm desvio padrão $(p=0,001739)$; B: Tempo de internamento dos doentes submetidos a ATA. Os valores são apresentados como média \pm desvio padrão $(p=0,00359)$. 
Com a implementação da recuperação pós-operatória de sangue verificou-se ainda uma diminuição na percentagem de doentes que receberam pelo menos uma unidade de $\mathrm{CE}$, no período pós-operatório. Após a implementação da estratégia em análise apenas $29 \%$ dos doentes submetidos a ATJ e $38 \%$ dos submetidos a ATA receberam transfusão de CE, em oposição aos $68 \%$ e $59 \%$ observados antes da implementação da recuperação pós-operatória de sangue nos doentes submetidos a ATJ e ATA, respetivamente (Fig. 2). Segundo a literatura, quando não é usada qualquer estratégia para reduzir a necessidade de transfusão de CE, $20 \%$ a $50 \%$ dos doentes submetidos a ATJ6 e $30 \%$ a $40 \%$ dos doentes submetidos a ATA recebem transfusão de CE, 8 valores superiores aos verificados no nosso estudo (Fig. 2). Estas diferenças podem ser justificadas pela variabilidade de critérios na definição dos limites de transfusão observados entre os hospitais. ${ }^{32}$ No entanto, a literatura é consensual no que diz respeito à redução da percentagem de doentes que recebem transfusão de $\mathrm{CE}$, no período pós-operatório, com o uso da recuperação pós-operatória de sangue. ${ }^{8,28,30,33}$ No estudo de Kumar et al, ${ }^{28}$ em 100 doentes submetidos a ATJ e em que foi utilizada a recuperação pós-operatória de sangue, apenas $11 \%$ receberam CE. Moonen et $\mathrm{al}^{31}$ analisaram 438 doentes submetidos a ATJ e ATA com recuperação pós-operatória de sangue, e constataram que apenas $5,1 \%$ dos doentes submetidos a ATJ e $8,4 \%$ dos doentes submetidos a ATA necessitaram de transfusão de CE. Nestes estudos a percentagem de doentes que recebeu transfusão de CE foi menor do que a observada no nosso estudo. Estas diferenças podem ser justificadas pela forma como os dispositivos de recuperação pós-operatória de sangue são usados, na experiencia dos profissionais de saúde no uso da estratégia, na duração de tempo considerada para a recuperação de sangue do local cirúrgico e no volume de sangue reinfundido nos doentes. A redução da necessidade de componentes de sangue homólogo tem a vantagem de evitar os efeitos adversos associados à transfusão de componentes de sangue homólogo e poupar sangue homólogo, que como já foi referido é um recurso limitado e caro.

A implementação da recuperação pós-operatória de sangue nas ATJ e ATA também se traduziu em alterações estatisticamente significativas no tempo de internamento. Após a implementação da recuperação pós-operatória de sangue os doentes submetidos a ATJ e ATA ficaram internados em média 8,3 e 8,8 dias, respetivamente, enquan- to antes da implementação da referida estratégia os períodos de internamento tinham a duração média de 9,0 e 9,1 dias, respetivamente (Fig. 3). Estes resultados estão de acordo com dados publicados anteriormente, ${ }^{29,34}$ apesar de Smith et $\mathrm{al}^{8}$ não terem verificado diferenças significativas em doentes submetidos a ATA. Esta redução no tempo de internamento pode ser justificada por uma recuperação mais rápida, ou por redução do número de episódios infeciosos. ${ }^{29,34}$ Segundo Gharehbaghian et al, ${ }^{35}$ a reinfusão de sangue recuperado do local cirúrgico parece ativar a imunidade sistémica em doentes submetidos a ATJ, atendendo ao aumento de percursores de células natural killer e ao aumento da concentração de interferão $\gamma$, invertendo assim a imunossupressão associada ao trauma cirúrgico e à perda de sangue. A redução no tempo de internamento, com a implementação da recuperação pós-operatória de sangue, é indicativo de uma recuperação mais rápida por parte do paciente, o que suporta a segurança e boa qualidade do sangue recuperado do local cirúrgico e reinfundido depois de filtrado.

Alternativamente à recuperação pós-operatória de sangue existem outras estratégias que visam o mesmo objetivo, minimizar a necessidade transfusional, como a doação autóloga pré-operatória e a recuperação intra-operatória de sangue. No entanto, a recuperação pós-operatória de sangue parece ser a estratégia mais adequada às ATJ e ATA.

\section{CONCLUSÃO}

Os resultados do presente trabalho permitem concluir que a introdução da estratégia de recuperação pós-operatória de sangue, nas cirurgias ortopédicas ATJ e ATA, diminui significativamente a necessidade de transfusão de CE, reduzindo também o tempo de internamento. Estudos futuros de natureza prospetiva serão importantes para avaliar de que forma o tempo de recuperação do sangue do local cirúrgico e o volume de sangue reinfundido aos doentes podem ser otimizados.

\section{CONFLITO DE INTERESSES}

Os autores declaram não ter nenhum conflito de interesses relativamente ao presente artigo.

\section{FONTES DE FINANCIAMENTO}

Não existem fontes externas de financiamento para a realização deste artigo.

\section{REFERÊNCIAS}

1. Johanson NA, Litrenta J, Zampini JM, Kleinbart F, Goldman HM. Surgical treatment options in patients with impaired bone quality. Clin Orthop. 2011;469:2237-47

2. Kurtz SM, Ong KL, Schmier J, Zhao K, Mowat F, Lau E. Primary and revision arthroplasty surgery caseloads in the United States from 1990 to 2004. J Arthroplasty. 2009;24:195-203.

3. Singh VK, Singh PK, Javed S, Kumar K, Tomar J. Autologous transfusion of drain contents in elective primary knee arthroplasty: Its value and relevance. Blood Transfus. 2011;9:281-5.

4. Liu X, Zhang X, Chen Y, Wang Q, Jiang Y, Zeng B. Hidden Blood Loss After Total Hip Arthroplasty. J Arthroplasty. 2011;26:1100-5.
5. Tellisi N, Kakwani R, Hulse N, Abusitta G, Ashammakhi N, Wahab KA Autologous blood transfusion following total knee arthroplasty: is it always necessary? Int Orthop. 2006;30:412-4.

6. Muñoz M, Ariza D, Florez A, Campos A. Reinfusion drains reduce postoperative transfusion requirements after primary total knee replacement surgery. Transfus Med. 2008;18:269-71.

7. Bierbaum BE, Hill C, Callaghan JJ, Galante JO, Rubash HE, Tooms RE, et al. An analysis of blood management in patients having a total hip or knee arthroplasty. J Bone Joint Surg Am. 1999;81:2-10.

8. Smith LK, Williams DH, Langkamer VG. Post-operative blood salvage with autologous retransfusion in primary total hip replacement. J Bone 
Joint Surg Br. 2007:89:1092-7.

9. Marcucci C, Madjdpour C, Spahn DR. Allogeneic blood transfusions: Benefit, risks and clinical indications in countries with a low or high human development index. Br Med Bull. 2004;70:15-28.

10. Bihl F, Castelli D, Marincola F, Dodd RY, Brander C. Transfusion-transmitted infections. J Transl Med. 2007;5:25.

11. Zadzilka JD, Stulberg BN. Blood Conservation in Total Knee Arthroplasty: Hedging Your Bets. Semin Arthroplasty. 2011;22:150-2.

12. Squires JE. Risks of transfusion. South Med J. 2011;104:762-9.

13. Llewelyn CA, Hewitt PE, Knight RSG, Amar K, Cousens S, Mackenzie $\mathrm{J}$, et al. Possible transmission of variant Creutzfeldt-Jakob disease by blood transfusion. Lancet. 2004;363:417-21.

14. Sinha A, Sinha M, Burgert S. Reinfusion of drained blood as an alternative to homologous blood transfusion after total knee replacement. Int Orthop. 2001;25:257-9.

15. Goodnough LT, Shander A, Brecher ME. Transfusion medicine: Looking to the future. Lancet. 2003;361:161-9.

16. D'Aloia A. Strategies for reducing transfusions in major orthopaedic surgery. Blood Transfus. 2011;9:246-7.

17. Ferguson E, Prowse C, Townsend E, Spence A, Hilten JAV, Lowe K. Acceptability of blood and blood substitutes. J Intern Med. 2008;263:24455

18. Stulberg BN, Zadzilka JD. Blood Management Issues: Using Blood Management Strategies. J Arthroplasty. 2007;22:S95-8.

19. Pavesi M, Inghilleri G, Albano G, Ricci C, Gaeta M, Randelli F. A predictive model to reduce allogenic transfusions in primary total hip arthroplasty. Transfus Apher Sci. 2011;45:265-8.

20. Jeer PJS, Cossey AJ, Keene GCR. Haemoglobin levels following unicompartmental knee arthroplasty: Influence of transfusion practice and surgical approach. Knee. 2005;12:358-61.

21. Matsuda K, Nozawa M, Katsube S, Maezawa K, Kurosawa H. Reinfusion of unwashed salvaged blood after total knee arthroplasty in patients with rheumatoid arthritis. Int Orthop. 2009;33:1615-8.

22. Laranjeira H, Fernandes N, Ferreira R, Borges L. Recuperação pósoperatória de sangue como alternativa à transfusão homóloga na artroplastia total do joelho e na artroplastia total da anca. Rev Soc Port Anestesiol. 2012;21:8-17.

23. Kleinert K, Werner C, Mamisch-Saupe N, Kalberer F, Dora C. Closed suction drainage with or without re-transfusion of filtered shed blood does not offer advantages in primary non-cemented total hip replacement using a direct anterior approach. Arch Orthop Trauma Surg. 2012;132:131-6.

24. Horstmann WG, Slappendel R, Van Hellemondt GG, Castelein RM, Verheyen CCPM. Safety of retransfusion of filtered shed blood in 1819 patients after total hip or knee arthroplasty. Transfus Altern Transf Med. 2010;11:57-64.

25. Guan ZP, Jiang J, Lv HS, Wang N. Research on coagulation of unwashed shed blood after total knee replacement in Chinese patients. Artif Cells Blood Substit Immobil Biotechnol. 2008;36:51-62.

26. Helwig U, Schauß S, Berghold A, Ziervogel H. Coagulation Parameters After Retransfusion of Unwashed Blood. J Arthroplasty. 2006;21:385-91.

27. R Development Core Team. R: A language and environment for statistical computing. Viena: RDCT; 2012.

28. Kumar G, Von Arx OA, Pozo JL. Rate of blood loss over 48 hours following total knee replacement. Knee. 2005;12:307-9.

29. Newman JH, Bowers M, Murphy J. The clinical advantages of autologous transfusion. A randomised, controlled study after knee replacement. J Bone Joint Surg Br. 1997;79:630-2.

30. Athanasoulias V, Mavrogenis AF, Sdrenias CV, Mitsiokapa EA, Lourikas V, Papagelopoulos PJ, et al. Post-operative blood salvage and retransfusion in total hip and knee arthroplasty. J Int Med Res. 2007;35:268-75.

31. Moonen AFCM, Thomassen BJW, Van Os JJ, Verburg AD, Pilot P. Retransfusion of filtered shed blood in everyday orthopaedic practice. Transfus Med. 2008;18:355-9.

32. Barr PJ, Donnelly M, Cardwell C, Alam SS, Morris K, Parker M, et al. Drivers of Transfusion Decision Making and Quality of the Evidence in Orthopedic Surgery: A Systematic Review of the Literature. Transfus Med Rev. 2011;25:304-16.

33. Muñoz M, Cobos A, Campos A, Ariza D, Muñoz E, Gomez A. Post-operative unwashed shed blood transfusion does not modify the cellular immune response to surgery for total knee replacement. Acta Anaesthesiol Scand. 2006;50:443-50.

34. Muñoz M, Ariza D, Garcerán MJ, Gómez A, Campos A. Benefits of postoperative shed blood reinfusion in patients undergoing unilateral total knee replacement. Arch Orthop Trauma Surg. 2005;125:385-9.

35. Gharehbaghian A, Haque KMG, Truman C, Evans R, Morse R, Newman $\mathrm{J}$, et al. Effect of autologous salvaged blood on postoperative natural killer cell precursor frequency. Lancet. 2004;363:1025-30. 